\title{
Nobel Lecture: From spin waves to giant magnetoresistance and beyond ${ }^{*}$
}

\author{
Peter A. Grünberg \\ Institut für Festkörperforschung, Forschungszentrum Jülich, Germany
}

(Published 17 December 2008)

DOI: $10.1103 /$ RevModPhys.80.1531

\section{DISCOVERY OF LIGHT SCATTERING FROM DAMON-ESHBACH SURFACE SPIN WAVE}

The "Institute for Magnetism" within the department for solid-state physics at the research center in Jülich, Germany, which I joined in 1972, was founded in 1971 by Professor W. Zinn. The main research topic was the exploration of the model magnetic semiconductors $\mathrm{EuO}$ and EuS with Curie temperatures $T_{c}=60 \mathrm{~K}$ and $T_{c}$ $=17 \mathrm{~K}$, respectively. As I had been working with light scattering (LS) techniques before I came to Jülich, I was very much interested in the observation of spin waves in magnetic materials by means of LS.

LS can be performed with grating spectrometers, which is called Raman spectroscopy, and alternatively by Brillouin light scattering (BLS) spectroscopy. In the latter case, a Fabry-Pérot (FP) interferometer is used for the frequency analysis of the scattered light (see righthand side of Fig. 1). The central part consists of two FP mirrors whose distance is scanned during operation. BLS spectroscopy is used when the frequency shift of the scattered light is small (below $100 \mathrm{GHz}$ ), as expected for spin waves in ferromagnets. In the early 1970s, an interesting instrumental development took place in BLS, namely, the invention of the multipass operation, and later, the combination of two multipass interferometers in tandem. The inventor was Dr. J. A. Sandercock in Zürich. Since we had the opportunity to install a new laboratory, we decided in favor of BLS, initially using a single three-pass instrument as displayed on the righthand side of Fig. 1. With this, we started investigating spin waves in $\mathrm{EuO}$.

We indeed were able to find and identify the expected spin waves as shown by the peaks in Fig. 1 (marked green). Different intensities on the Stokes (S) and antiStokes (aS) side were known from other work to be due to the magneto-optic interaction of light with the spin waves.

The peaks marked red remained a puzzle for some time until good luck came to help us. Good luck in this case was a breakdown of the system, a repair and unintentional interchange of the leads when reconnecting the magnet to the power supply. To our surprise $\mathrm{S}$ and aS

\footnotetext{
*The 2007 Nobel Prize for Physics was shared by Albert Fert and Peter Grünberg. This paper is the text of the address given in conjunction with the award.
}

side were now reversed. To understand what this means, one has to know that classically $\mathrm{S}$ and aS scattering is related to the propagation direction of the observed mode, which is opposite for the two cases. This can be understood from the corresponding Doppler shift, which is to higher frequencies when the wave travels towards the observer and down when away from him. The position of the observer here would be the same as of the viewer in Fig. 1. The appearance of the red peak in the spectra on only either the $\mathrm{S}$ or the aS side can be explained by an unidirectional propagation of the corresponding spin wave along the surface of the sample. It can be reversed by reversing $B_{0}$ and $M$.

The unidirectional behavior of the wave can be understood on the basis of symmetry. For this, one has to know that axial vectors which appear in nature, such as $B$ and $M$ on the left-hand side of Fig. 1, reverse their sign under time inversion and so does the sense of the propagation of the surface wave as indicated. The upper and lower parts of Fig. 1, on the left-hand side therefore are linked by time inversion symmetry, which is valid without damping. Hence, the unidirectional behavior reflects the symmetry of the underlying system. Finally, the observed wave could be identified as the DamonEshbach (DE) surface mode known from theory and from microwave experiments.

From the magnetic parameters of $\mathrm{EuO}$, one predicts in the present case that the penetration depth of the DE mode will be a few $100 \AA$. Sample thickness $d$ is of the order of $\mathrm{mm}$. Therefore, for the present purpose, $\mathrm{EuO}$ is opaque. In this case, the wave traveling on the backside of the sample in the opposite direction to the wave on the front side cannot be seen in this experiment. BLS is then either $\mathrm{S}$ or aS but not both at the same time. Due to all of these unique features, the results of Fig. 1 have also been chosen as examples for current research in magnetism in a textbook on "Solid State Physics" (Ibach and Lüth, 1995, p. 186).

\section{BLS FROM SPIN WAVES IN SINGLE THIN MAGNETIC FILMS}

We stick to the geometry of Fig. 1 but with much smaller thickness $d$, of the order of $200 \AA$ A. As material, we chose Fe. Bulk modes now split into a bunch of standing modes and shift to higher frequencies. In the example in Fig. 2, only the DE mode and the first stand- 


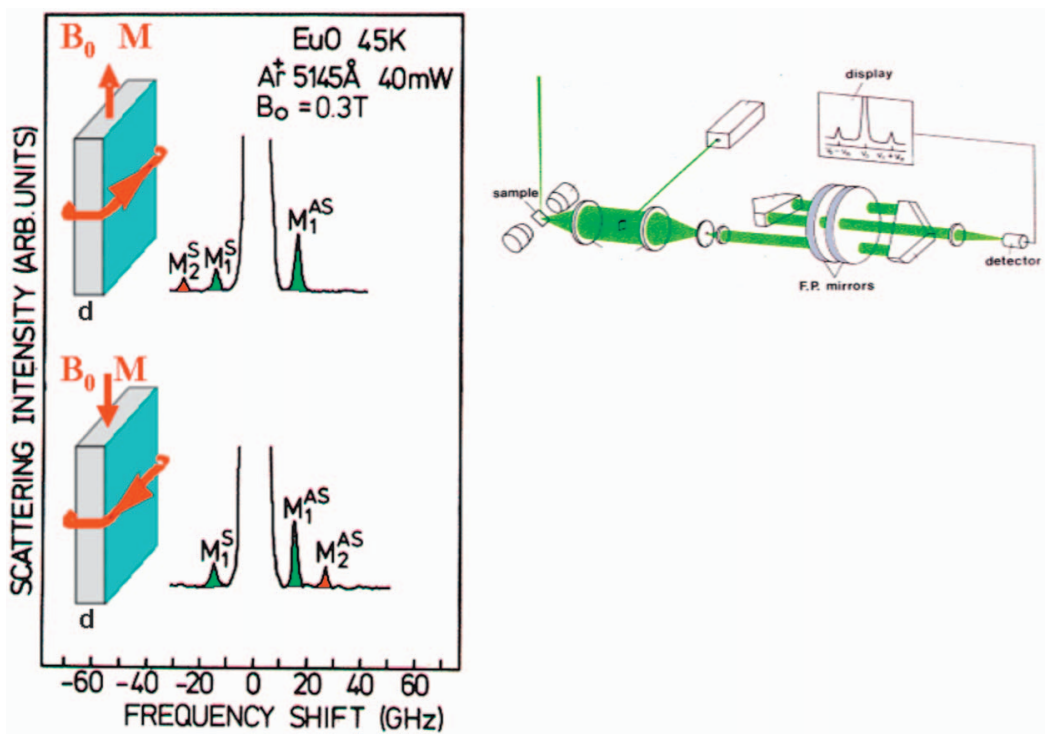

FIG. 1. (Color) Right: Schematic of a Brillouin light scattering (BLS) spectrometer used for the detection of spin waves. Spectra of scattering from bulk spin waves (marked with green) and DE surface spin waves (red) are seen on the left. For the upper and lower parts on the left, the field $B_{0}$ and magnetization $M$ have been reversed. From Grünberg and Metawe, 1977.

ing mode are within the observable frequency range. The mode patterns (left) represent amplitude distributions, where the local precession is generally elliptical. $\mathrm{DE}$ modes with given propagation direction have finite amplitudes on both surfaces and therefore both directions are seen as $\mathrm{S}$ and aS scattering. As in Fig. 1, the sample surface from which most of the scattering is observed is shown in turquoise.

\section{DIPOLAR COUPLED DE MODES IN MAGNETIC DOUBLE LAYERS WITH PARALLEL AND ANTIPARALLEL MAGNETIC ALIGNMENT}

Next, we consider magnetic double layers (sometimes also called trilayers) as illustrated on the left-hand side of Fig. 3 (Grünberg, 1989). There are four magnetic surfaces, each carrying a DE wave, which interact via the oscillating stray fields that they produce. The calculation proceeds by using the Landau-Lifshitz equation after linearization and by applying the boundary conditions following from Maxwell's equations. The middle section of the figure displays the result for the mode frequencies as a function of the interlayer thickness $d_{0}$ multiplied by the value of the wave vector $q$ of the wave. $q$ is determined by the geometry of the BLS experiment, including the wavelength of the laser light used. A typical value is $q=1.73 \times 10^{-2} \mathrm{~nm}^{-1}$. Hence $q d_{0}=1$ corresponds to $d_{0}=58 \mathrm{~nm}$. This is the decay length of the DE waves away from the surface.

For the parallel alignment, the calculation predicts a symmetric graph, i.e., the same frequencies for both signs of $q$. For the antialignment, the predicted branches are not symmetric as also revealed by the experiment. Figure 3 displays a few more details of the nature of these modes. The middle part reveals that the upper branches $1+$ and 1- are characterized by an in-phase precession of the magnetizations $M$, whereas for the lower branches $2+$ and $2-$ they are out of phase by $180^{\circ}$. In the middle part on the left-hand side, this aspect is again illustrated by showing the amplitude distribution inside a cross section, where the DE attenuation has been neglected. Note the phase jump by $180^{\circ}$ in the middle part of the structure.

\section{DOUBLE LAYER WITH THE INCLUSION OF INTERLAYER EXCHANGE}

Qualitatively, the behavior of the dipolar modes due to the inclusion of ferromagnetic $(F)$ interlayer exchange coupling (IEC) is easy to predict. This is because the result of full $\mathrm{F}$ coupling is a single film of which we already know the result as displayed in Fig. 2. Why, in Fig. 4, is it the lower dipolar branch from which the first standing mode evolves and not the upper one? It becomes clear from the nature of the modes as displayed in the middle, upper part of Fig. 3 and has to do with the conservation of the symmetry character. When IEC is "turned on," the amplitude pattern with the $180^{\circ}$ phase jump is changed into the first standing mode under conservation of parity. Note that a $180^{\circ}$ phase jump would

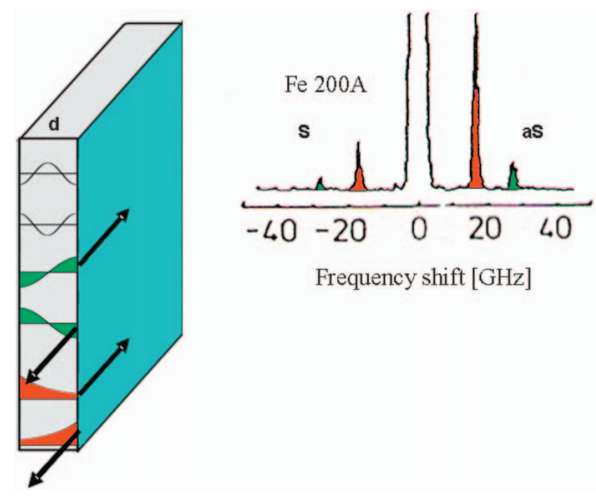

FIG. 2. (Color) DE surface spin wave and first standing spin wave of a single thin film of Fe and its experimental observation by means of BLS. Opposite propagation directions (black arrows) correspond to $\mathrm{S}$ and aS scattering. Higher standing waves as indicated in the upper part on the left-hand side are beyond the observed frequency range (Grünberg, 1989). 

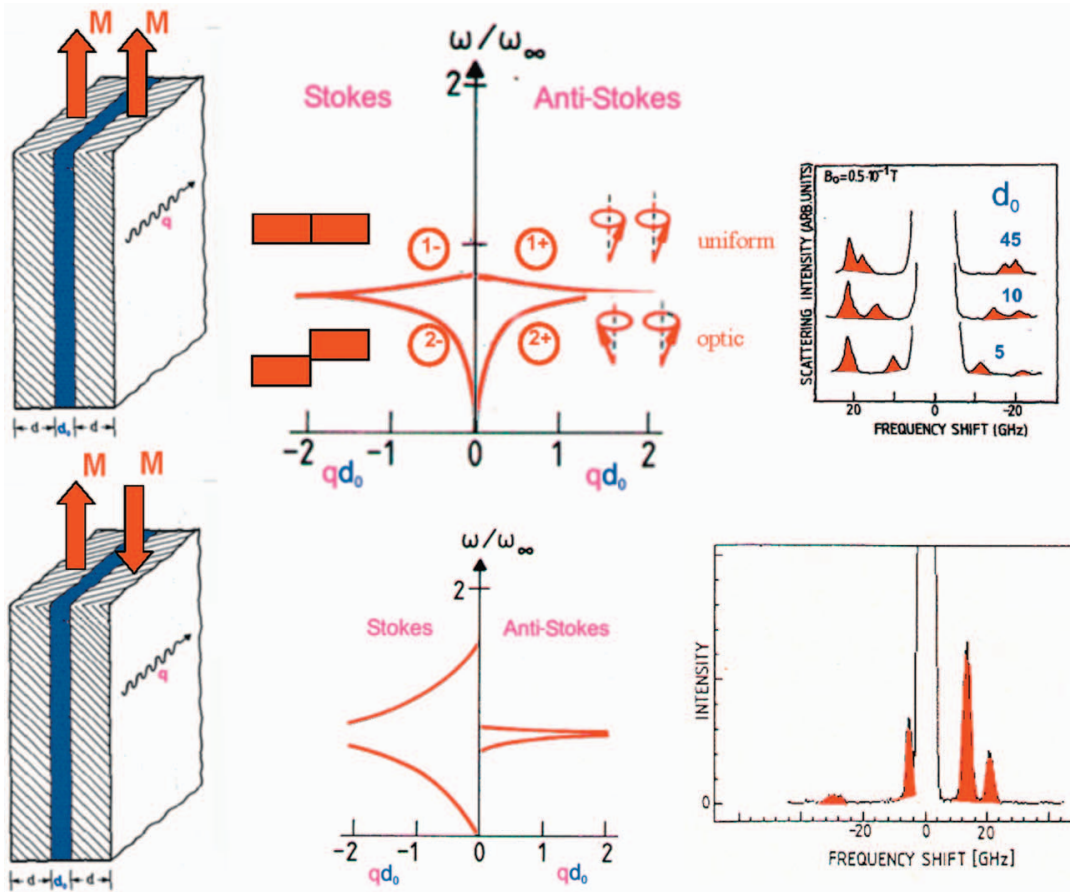

FIG. 3. (Color) Coupled DE modes in magnetic double layers for parallel (upper part) and antiparallel (lower part) alignment of the magnetization $M$ (Grünberg, 1989). Calculation in the dipolar limit. For the spectrum in antiparallel alignment, a sample showing AF coupling (to be discussed later) was used. Peak positions are mainly due to the dipolar interaction with small corrections due to exchange coupling.

generate a very high exchange energy which is avoided by the formation of a node (as in Fig. 2).

In Fig. 3, we looked at the possibility of an antiparallel alignment and one way of achieving it would be antiferromagnetic (AF) interlayer exchange coupling. If we want to recognize this type of coupling from the behavior of the spin waves, what do we expect? At this point, it is important to mention that most of our experiments are performed in saturation. Hence, regardless of whether the coupling is F or AF, we would apply a high enough field for the BLS experiment to get parallel alignment (with an important exception as seen below). This has the advantage of a well-defined experimental situation. The upper branch in the case of magnetic saturation is given by the uniform precession, i.e., total moment and therefore independent of IEC.

For the lower branch, one can argue as follows. For magnetizations aligned parallel, an external field as well as ferromagnetic IEC should increase the restoring force and hence also the spin-wave frequency. Opposite to this, AF type IEC should weaken the effect of the external field and thus decrease the frequency. In the present case, the dipolar interaction works in a similar way. Hence, from these simple arguments one expects the frequency of the lower branch to be further decreased for antiferromagnetic IEC and to be increased as shown in Fig. 4 for ferromagnetic IEC. On the other hand, if IEC is antiferromagnetic, then for small enough external fields we should obtain antialignment. In this case, the branches presented in the lower part of Fig. 3 could be used to identify the antialignment.

The concept just presented was later used and worked out in more detail by Barnas and Grünberg (1989) and by Cochran and Dutcher (1988). From the latter work, we have adopted the term "uniform mode" for the inphase precession and "optic mode" for the $180^{\circ}$ out-of-

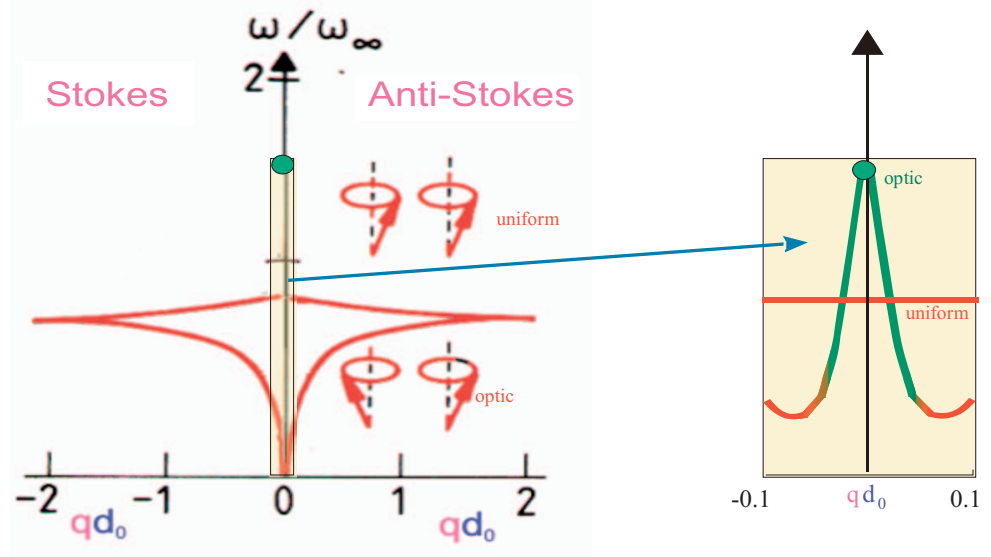

Interlayer dipole dipole interaction only
Including interlayer exchange Here assumed ferromagnetic
FIG. 4. (Color) The expected effect of ferromagnetic interlayer exchange on the branches representing dipolar coupling (red). In the limit of full exchange for $d_{0}=0$, the lower branch is replaced by the green dot representing the first standing mode of the combined single film. The general behavior for small $d_{0}$ is shown on the right-hand side. For the lower branch, there is a transition from dipolar dominated (red) to interlayer exchange dominated (green). The upper dipolar branch becomes the DE mode of the combined film. In the displayed region of small $d_{0}$, its frequency is practically constant (horizontal red line). 

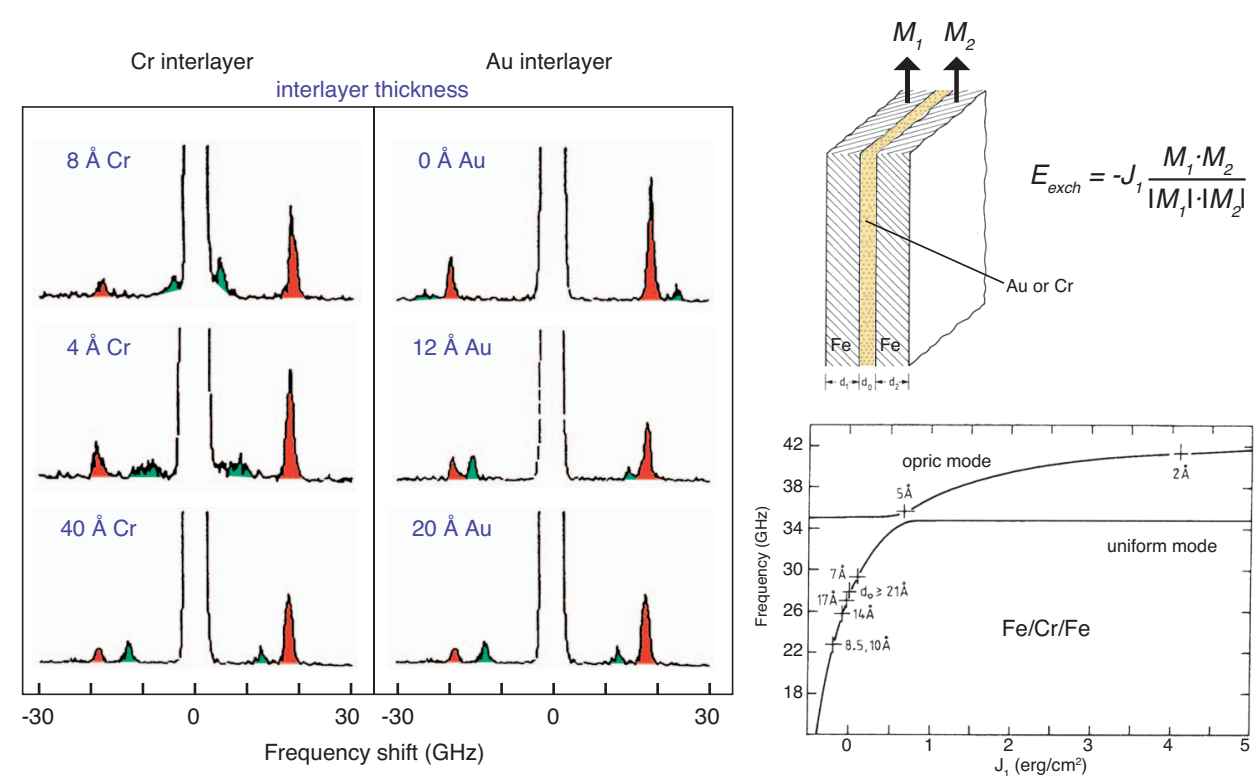

\begin{abstract}
FIG. 5. (Color) Left-hand side: Representative BLS spectra from spin waves in $\mathrm{Fe} / X / \mathrm{Fe}$, where $\mathrm{X}=\mathrm{Cr}$ and $\mathrm{Au}$ (Grünberg et al., 1986). The plot on the right-hand side displays the mode frequencies as a function of the interlayer exchange parameter $J_{1}$ (Barnas and Grünberg, 1989), where the theory is represented by the solid lines.
\end{abstract}

phase precession. The principle was also used by other groups for the evaluation of exchange coupling from microwave experiments.

Due to the insight just described, we became aware in the early 1980s that we had a powerful tool in our hands, which could be used to investigate a long-standing open question concerning coupling phenomena in layered magnetic structures. At that time, it was suggested that ferromagnetic coupling could be caused by pinholes and ferromagnetic bridges across the interlayer. As Néel showed, it could also be due to meandering interlayers. This effect was therefore called orange peel or Néeltype coupling. Antiferromagnetic-type coupling could possibly occur due to edge effects, such as flux closure. Real intrinsic types of interlayer coupling, similar to the Ruderman-Kittel-Kasuya-Yosida (RKKY) interaction in dilute magnetic alloys, was not yet known. The situation was further hampered by insufficient sample preparation. In our first attempt to explore coupling and its dependence on the interlayer material and thickness we only found ferromagnetic-type coupling-presumably resulting from pinholes.

For various reasons, we now concentrated on $\mathrm{Cr}$ interlayers. First, $\mathrm{Cr}$ by itself is an antiferromagnet. Based on its magnetic structure for thin enough interlayers, one expects the coupling to oscillate between $\mathrm{F}$ and $\mathrm{AF}$ with a period of two monolayers (MLs). Second, $\mathrm{Cr}$ matches Fe very nicely in both its crystallographic and thermodynamic properties, which is important for the growth. $\mathrm{Fe} / \mathrm{Cr} / \mathrm{Fe}$ structures therefore appeared to be most promising.

\section{DISCOVERY OF ANTIFERROMAGNETIC (AF) COUPLING}

In this situation, I was very fortunate to have the opportunity to take sabbatical leave and go to Argonne National Laboratories in the U.S. In the team around M. Brodsky in Materials Sciences, I found a group with expertise in preparing $\mathrm{Fe} / \mathrm{Cr} / \mathrm{Fe}$ samples on substrates of cleaved rocksalt. For comparison, we also prepared $\mathrm{Fe} / \mathrm{Au} / \mathrm{Fe}$ samples.

Spectra from both systems in strong enough fields for full saturation of the samples are displayed on the lefthand side of Fig. 5. The spectrum with $d_{0}=0$ represents the fully F-coupled limit as in Fig. 3. The two spectra at the bottom represent pure dipolar coupling. From the $\mathrm{Fe} / \mathrm{Au} / \mathrm{Fe}$ data and comparison with Fig. 4, we concluded that the coupling for the Au interlayers is ferromagnetic. However, by looking at the spectrum in the upper corner on the left-hand side for the $\mathrm{Cr}$ interlayers, the reader might share with me the sensation I had at that time when I viewed a BLS spectrum representing antiferromagnetic IEC for the first time.

In a phenomenological approach, IEC is treated via the associated aerial energy density $E_{\text {exch }}$, as quoted in the figure. As already discussed, on physical grounds, the optic mode increases its frequency for $\mathrm{F}$ coupling $\left(J_{1}>0\right)$ and decreases it for AF coupling $\left(J_{1}<0\right)$.

Figure 6 displays the effect of $\mathrm{F}$ and AF coupling on the remagnetization (so-called hysteresis) curve. Plotted is the magneto-optic Kerr effect (MOKE) signal, which is roughly proportional to the magnetization $M$, vs the external field $B_{0}$. There is a strong shearing for the $\mathrm{Fe} / \mathrm{Cr} / \mathrm{Fe}$ sample, which was not observed for the other $(\mathrm{Fe} / \mathrm{Au} / \mathrm{Fe})$ sample, which displays the remagnetization of an uncoupled or F-coupled sample with small coercivity. F coupled and uncoupled cannot be distinguished. Furthermore, one has to consider that shearing of hysteresis curves is not very unique. It is a phenomenon that occurs very often and can have many causes. The use of more sophisticated tools like BLS is not a luxury for a first observation. Note also that the information given by the MOKE curve is assisted by BLS spectra to make sure there is AF alignment at these points. However, once it is certain that shearing comes from AF coupling, one might as well employ this simpler method to obtain large amounts of data. 


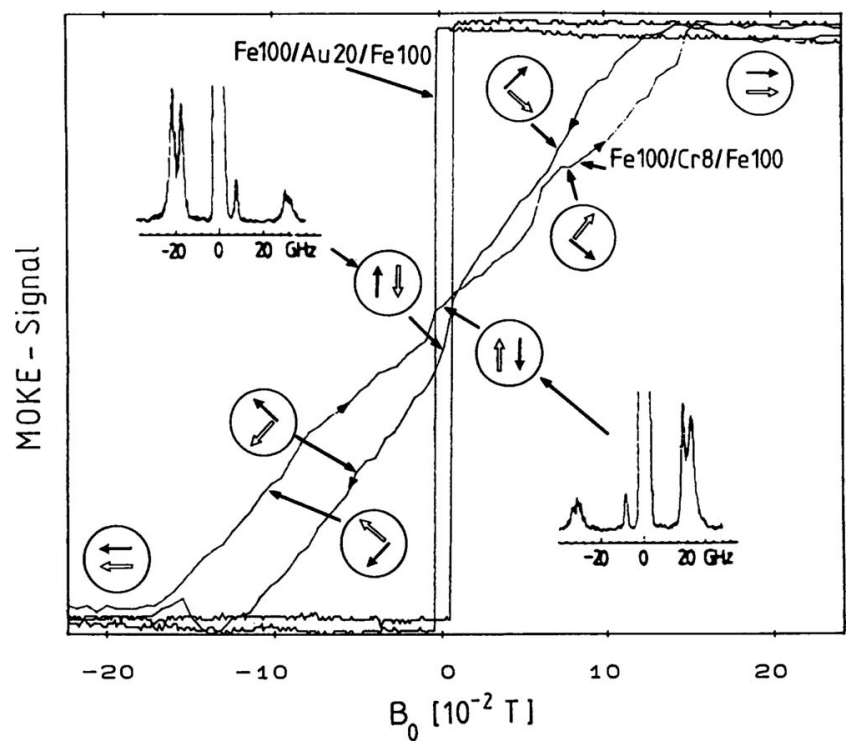

FIG. 6. Remagnetization curves of samples with F coupling $(\mathrm{Fe} / \mathrm{Au} / \mathrm{Fe})$ and $\mathrm{AF}$ coupling $(\mathrm{Fe} / \mathrm{Cr} / \mathrm{Fe})$.

In Fig. 7, we see the result of a more extended investigation of the coupling represented by mode frequencies as a function of the $\mathrm{Cr}$ interlayer thickness given in Angstroms. This is the first rather complete result obtained in May 1985, and is directly copied from the laboratory book. The minima of the lower branch indicate AF coupling in that range. The second shallower minimum initially appeared to be not sufficiently reproducible and was not communicated in our first publication. Furthermore, we were not very happy with the $\mathrm{NaCl}$ substrates because of their sensitivity to humidity. Since meanwhile epipolished GaAs had become available, we switched to this substrate material. Finally, AF coupling could reproducibly be observed where the result of Fig. 7 seemed generic instead of the expected period of 2 ML. However years later due to improved growth, this and other theoretically expected features caused by the noncommensurate spin density wave of $\mathrm{Cr}$ could be observed (Unguris et al., 1991).

In 1986, when we published our results (Grünberg et al., 1986), there were two other publications on interlayer exchange coupling, namely, of Dy and of Gd across $\mathrm{Y}$ interlayers observed by means of neutron scattering [cited in Bürgler et al. (2001)]. For Gd interlayers, it was reported to be oscillatory, as indicated by Fig. 7 and for many other examples, which followed.

\section{DISCOVERY OF GIANT MAGNETORESISTANCE}

Sir Nevil Mott proposed a two-current model for the description of the electrical resistivity of magnetic alloys. This model is based on the fact that the resistivity is due to electron scattering. In magnetic materials, this is dependent on spin orientation. Due to the quantummechanical spatial quantization, this orientation is only parallel or antiparallel with respect to the local magnetization. As spin flip processes seldom occur, each of the two orientations defines a current.

With this picture, one expects that there would be a strong resistivity change if one could manage to change the direction of the local magnetization within the mean-free path of the electrons or on an even shorter scale. In a case where the scattering rates are different, there is a better chance that the total scattering rate is increased. As mean-free paths are of the order of $10 \mathrm{~nm}$, the $1 \mathrm{~nm}$ thickness by which the magnetic layers are separated in the coupled structures perfectly fulfills this condition. We expect that if the relative orientation of the magnetizations changes from parallel to antiparallel in a structure such as in Fig. 8, left-hand side, the resistivity for the current will increase.

In order to have more experimental possibilities available, we used samples grown epitaxially on (110) oriented GaAs. The film plane was parallel to a (110) atomic plane and had an easy (EA) and a hard (HA) axis. For the thickness $d$ of the individual Fe films, we chose $d=12 \mathrm{~nm}$. The $C r$ thickness was $d_{0}=1 \mathrm{~nm}$, which corresponds to the first maximum of AF coupling. As a reference sample, we also made a single Fe film with thickness $d=25 \mathrm{~nm}$ in order to measure the anisotropic magnetoresistance (AMR) effect for comparison. Laterally, the samples had the shape of a long strip with contacts at both ends.

In Figs. 8(a) and 8(b) we can see the MOKE hysteresis loops from the double layers with AF coupling for $B_{0}$ along the EA and HA. The directions of the magnetization are indicated by the encircled pairs of arrows. This information is obtained from the MOKE intensities and
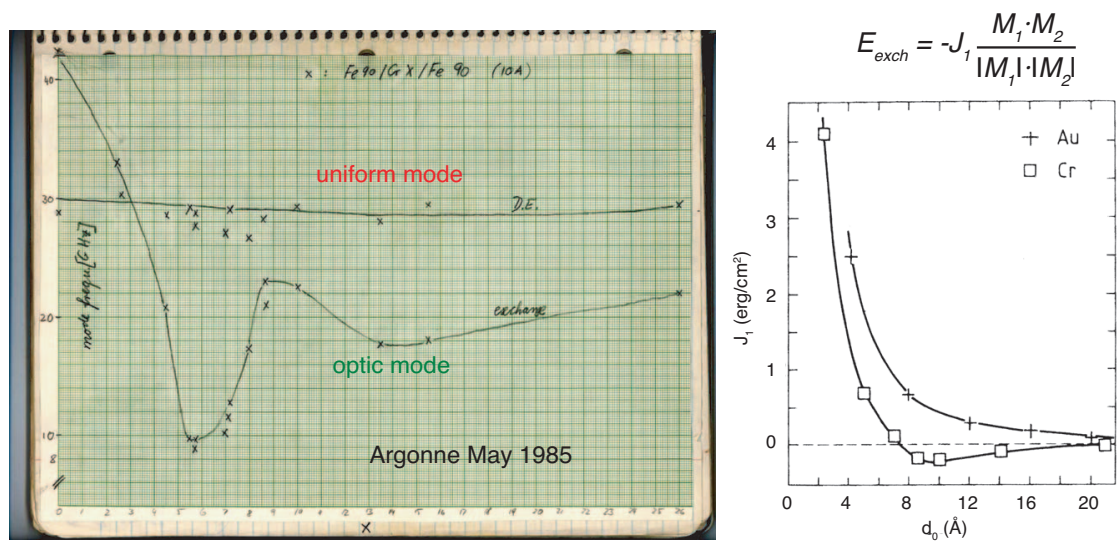

Rev. Mod. Phys., Vol. 80, No. 4, October-December 2008
FIG. 7. (Color) Left-hand side: First measurement of coupling for a wider range of $\mathrm{Cr}$ thicknesses, given in terms of spin wave frequencies. Note that the DE mode stays almost constant whereas the optic (or exchange) mode shows two minima, indicative of AF coupling. Righthand side: Evaluation of the frequencies for another sample not showing the second minimum, with respect to the coupling parameter $J_{1}$. 

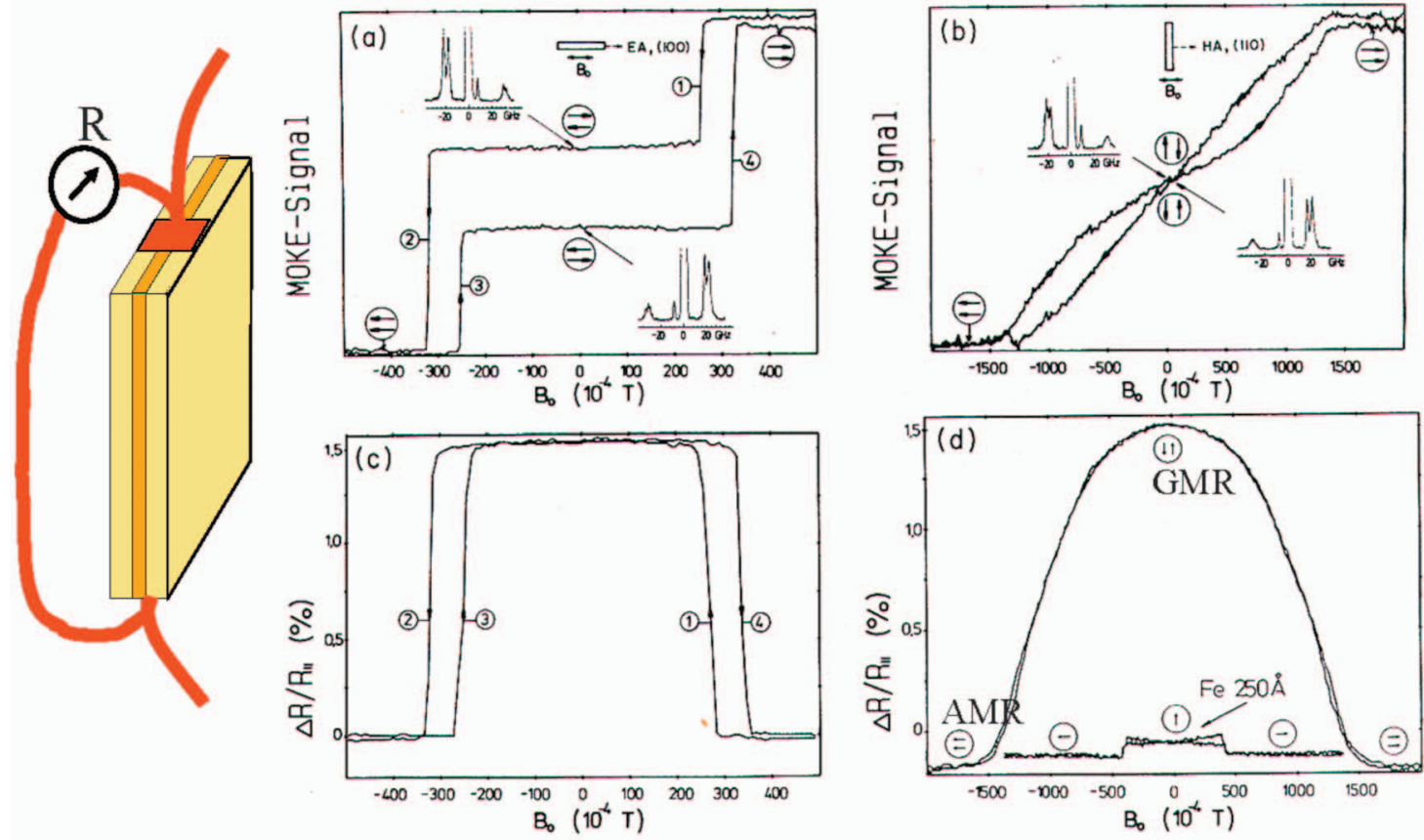

FIG. 8. (Color) (a) and (b) MOKE curves and BLS spectra in order to identify magnetic alignment as shown by arrows. (c) and (d) Relative resistance change corresponding to alignment (Binasch et al., 1989).

the displayed LS spectra. As an example, we discuss the hysteresis loop shown in Fig. 8(a) in more detail. The field $B_{0}$ is applied along the EA, which is the long axis of the strip. It is clear that for a large enough $B_{0}$, the samples saturate in the field direction (parallel alignment). If we start with parallel alignment in the positive field direction and reduce $B_{0}$ then at a certain, but still positive value of $B_{0}$, the magnetization of one film reverses via domain-wall motion (point 1 ). Hence, in small fields we have antiparallel alignment. In a negative field, at point 2 , the other film also reverses, and we have saturation. Points 3 and 4 mark the magnetization reversals when $B_{0}$ is scanned back.

Magnetoresistance (MR) traces are shown in Figs. 8(c) and $8(\mathrm{~d})$. Here we have plotted the relative change of the resistivity $\left(R-R_{\uparrow \uparrow}\right) / R_{\uparrow \uparrow}$ as we scan through the hysteresis loop. $R_{\uparrow \uparrow}$ is the resistivity for saturation along the EA. In Fig. 8(d), we also show the MR of the single Fe film, taken for $B_{0}$ along the HA. Since for large $B_{0}$, the magnetization is along [110] and for small $B_{0}$, along [100], the maximum change of $R$ seen in this trace displays the anisotropic $\mathrm{MR}=\left(R_{\infty}-R_{\|}\right) / R_{\|}$of a 25 -nm-thick Fe film. The value of $-0.13 \%$ is in reasonable agreement with the literature value of $-0.2 \%$. For the single film and $B_{0}$ along the EA, there was no measurable MR effect. The reason for this is clear; the magnetization always stays aligned parallel to the EA and magnetization reversal takes place via domain-wall motion only. In Fig. 8(d) for the $\mathrm{Fe}-\mathrm{Cr}-\mathrm{Fe}$ film, we have an MR effect due to both the anisotropic effect (negative values) and antiparallel alignment (positive values). The zero level of this plot is defined by $R=R_{\|}$.

At the time of the discovery of GMR, it was well known that leading computer companies planned to de- velop AMR so it could be used for read heads in hard disk drives (HDDs). The comparison between AMR and the new effect (later, the term GMR was widely accepted) encouraged us to file a patent for using GMR in HDD.

In Fig. 9 (Fert et al., 1995), we can see a comparison of the measurements using a multilayer as in Orsay and a double layer as in Jülich. The value for the multilayer of around $80 \%$ obtained in Orsay, which led to the term "giant" appears to have been surpassed by far by the TMR value of $500 \%$ for systems with $\mathrm{MgO}$ barriers. However, one has to be careful. There seems to be an important difference between dealing with insulators, semiconductors and metals. The magnetic semiconductor EuS, for example, changes its resistivity below its Curie point by many orders of magnitude. If we restrict the discussion to purely metallic systems, which has also some important implications for applications, the term giant is still justified.

The value $1.5 \%$ for the double layer displayed in Fig. 9(b) is even smaller but still large compared to the AMR effect of Fe (trace at the bottom). In Fig. 8, the effect
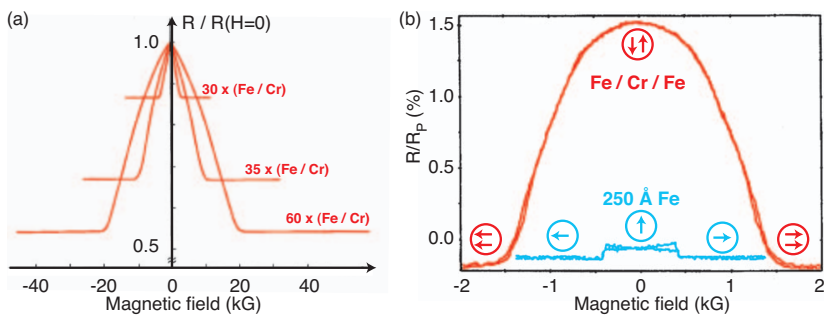

FIG. 9. (Color) GMR effect in (a) a multilayer and (b) a double layer of $\mathrm{Fe}$ interspaced by $\mathrm{Cr}$. In (b) the AMR of a 250 - $\AA$-thick film of $\mathrm{Fe}$ is also shown for comparison. 
(b)

(a)

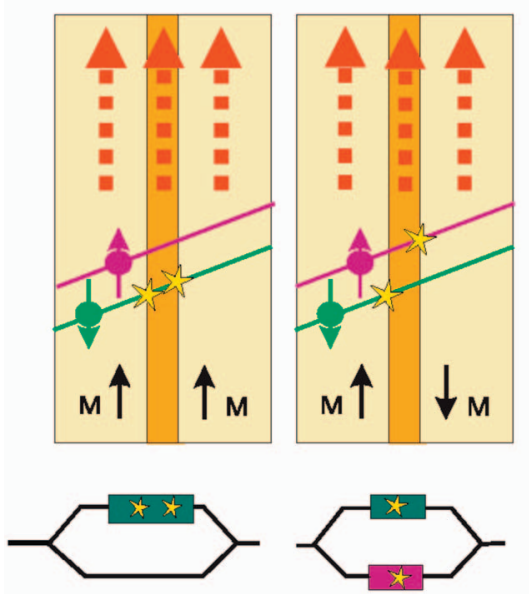

FIG. 10. (Color) (a) Explanation of the GMR effect: spin-dependent electron scattering and redistribution of scattering events upon antialignment of magnetizations. The antiparallel alignment can be obtained by antiferromagnetic coupling, like in Fig. 9, or due to hysteresis effects, like in (b). was measured using various configurations of external field relative to the axis of the crystal anisotropy to make sure that it really is due to the relative orientation of the magnetizations. The low values of the GMR effect as seen in Figs. 8 and 9 is not a result of minor sample quality but is typical for the chosen film thicknesses.

GMR can be observed in the current in plane (CIP) and current perpendicular plane (CPP) geometry. The conventional geometry is CIP and is for most sensor applications (see below). The CPP configuration is expected to be of interest for future sensor designs.

As was already suggested in the first paper by Albert Fert and his group and later established by numerical evaluations of experiments (Barnas et al., 1990) the microscopic explanation for the GMR effect is a dependence of the scattering rates on the orientation (parallel or antiparallel) of the electron spins with respect to the local magnetization.

We now consider the situation in Fig. 10(a). Two structures are shown, one with parallel and the other with antiparallel magnetization alignment. In an ideal situation, we assume that electrons with spin parallel to the local magnetization in their random walk are not scattered. In the figure, only one passage from the left to the right surface is displayed as representative for the entire motion. In this ideal picture, the electrons, which are not scattered, would cause a short circuit. This short circuit is removed when one magnetization is turned round and the electron with spin up enters this layer and finds its spin to be opposite to the local magnetization. The equivalent circuits for the various cases are shown at the bottom and display the increase in the resistivity due to the removal of the short circuit. In reality, of course, both types of electrons are scattered. It is sufficient that one sort of spin is scattered more than the other for the resistivity to increase due to the antiparallel magnetization alignment. From symmetry for the CIP configuration, spin-dependent interface reflectivity does not contribute to the effect. The reason is that there is no change in momentum in the direction of the current due to translational symmetry during reflection at interfaces. This is different in the CPP geometry, where both spindependent scattering as well as spin-dependent reflectivity contribute.

The largest difference in resistivity occurs when an $\mathrm{AF}$ alignment is changed by an applied field into a $\mathrm{F}$ alignment. The AF alignment can be provided by $\mathrm{AF}$ interlayer exchange as for the samples in Fig. 9 or by different coercivities of successive magnetic layers, in particular by pinning the magnetization using an antiferromagnetic material in direct contact, known as "exchange biasing." If GMR is obtained via one of the latter methods and not via AF interlayer coupling, usually the term "spin-valve system" is used in the literature, although there is no difference concerning the mechanism of the GMR effect. Such a case is displayed in Fig. 10(b) [see also the review article in Fert et al. (1995)]. The sample is a $\mathrm{Co} / \mathrm{Au} / \mathrm{Co}$ layered structure with one of the Co films deposited directly onto the GaAs substrate. Since this Co film is more strained than the one prepared on the $\mathrm{Au}$ interlayer, it has a higher coercivity. During magnetization reversal of the whole structure, it reverses later than the other, resulting in a small field range with antiparallel alignment. In Fig. 10(b) upper panel, this range is marked by antiparallel arrows. The curve in Fig. 10(b) lower panel shows the associated electrical resistance $R(H)$ and its increase due to the antiparallel alignment. As will be seen in the section on applications, the two different methods for obtaining antiparallel alignment lead to two different concepts for sensors based on GMR.

\section{GMR: THE CAMLEY-BARNAS MODEL}

At the time when the GMR effect was discovered, it was a coincidence that I had two visiting scientists in my team at Jülich, Jozef Barnas from Poznan in Poland and Bob Camley from Colorado Springs in the USA. Both are theoreticians whom I had been working with years before on spin waves. Together, they worked out a method for the description of GMR by means of suit- 


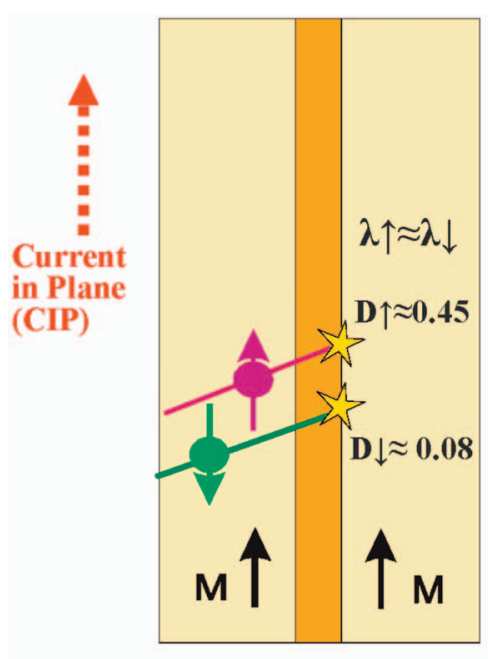

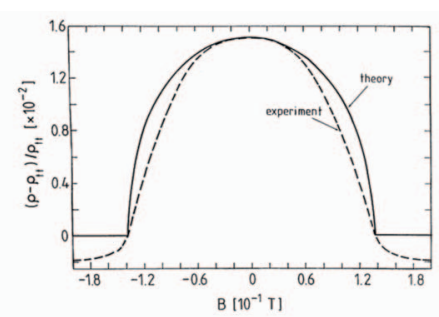

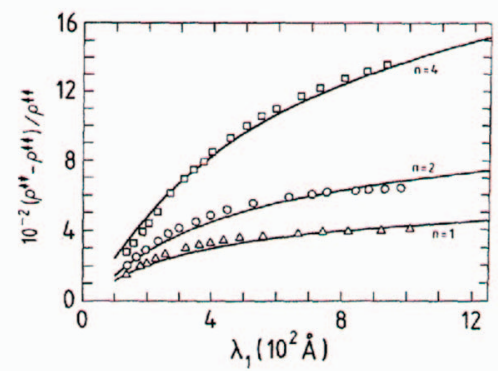

FIG. 11. (Color) Comparison of theory and experiment within the Camley-Barnas model. Top right: Resistance change vs external field assuming AF coupling and cubic anisotropy. Bottom right: Resistance change vs mean free path for multilayered structures ( $n=$ number of Cr layers). Left: Asymmetry of diffusive scattering parameters given by $D_{\uparrow} \approx 0.45$ and $D_{\downarrow} \approx 0.08$. able parameters. Their method is based on Boltzmann's diffusion equation within the relaxation time approximation and soon became known as the Camley-Barnas model Camley and Barnas, 1989, see Fig. 11.

\section{CIMS}

Current-induced magnetic switching (CIMS) can be understood as an inverse GMR effect. In a trilayer structure, one film is prepared with a fixed magnetization $M_{\text {fixed }}$ and the other with a magnetization, which can be easily rotated $\left(M_{\text {free }}\right)$. Using electron beam lithography, the samples are prepared in such a way that a strong current can be applied perpendicular to the sample plane. Then it can be shown that-no matter what the initial conditions are-when the current is such that the electrons flow from the film with $M_{\text {fixed }}$ to the film with $M_{\text {free }}, M_{\text {free }}$ switches into the parallel orientation. When the current is in the opposite direction $M_{\text {free }}$ switches into the antiparallel orientation. The effect was predicted by Slonczewski and Berger and observed by Katine et al. for the first time. References to these works are given in Grünberg et al. (2007), which also shows an example for "inverse" CIMS, where the role of the two current directions is interchanged.
Figure 12 displays an example for CIMS obtained recently in Jülich from epitaxial samples of $\mathrm{Fe} / \mathrm{Ag} / \mathrm{Fe}$ with fourfold cubic in-plane anisotropy. The curves with the overall $U$ shape display the resistivity of the sample in CPP geometry. The strong increase on both sides is due to the heating of the sample by the strong current. The various steps in the curves are due to switching between the various configurations as indicated and the associated change in the CPP-GMR effect. The shamrock patterns in the middle part display the cubic anisotropy. Magnetizations always switch between easy axes indicated by minima in the polar plot.

\section{APPLICATIONS}

We now come to applications of the effects discussed. One can safely say that GMR has received much attention due to its impact on magnetic storage, in particular in hard disk drives (HDDs). Since the introduction of GMR-type sensors as reading elements, in around 1997, storage capacities have increased approximately 100 times. The introduction of GMR sensors into HDDs was particularly fast because magnetoresistive sensors based on the anisotropic magnetoresistive effect (AMR) had paved the way. Thanks to the fact that GMR is at least

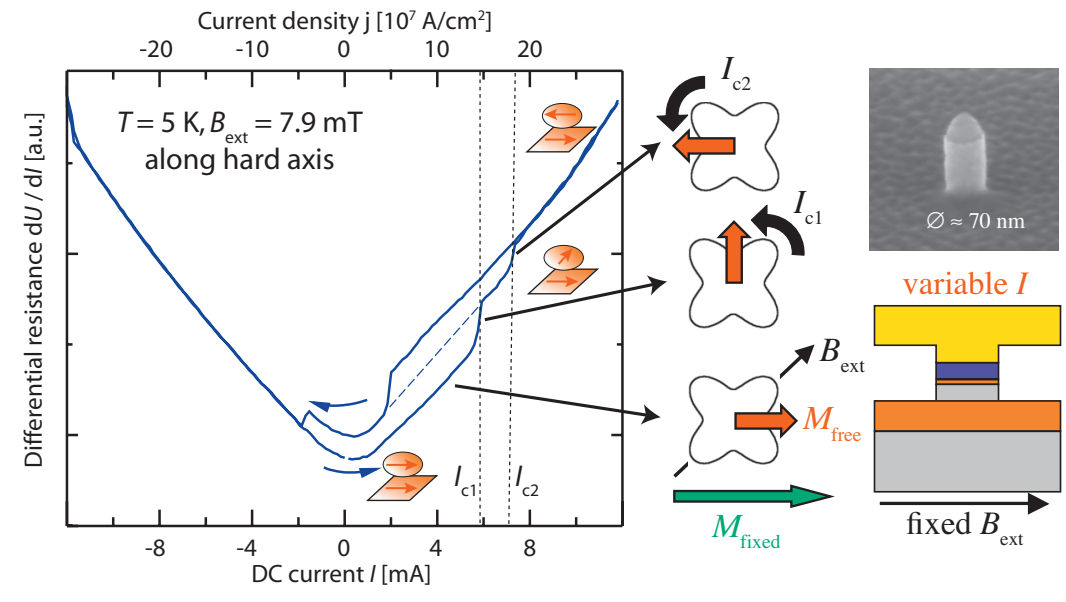

FIG. 12. (Color) Two-step CIMS in $\mathrm{Fe} / \mathrm{Ag} / \mathrm{Fe}$. Four energetically nearly identical states give rise to two-step switching (Lehndorff et al., 2007). 


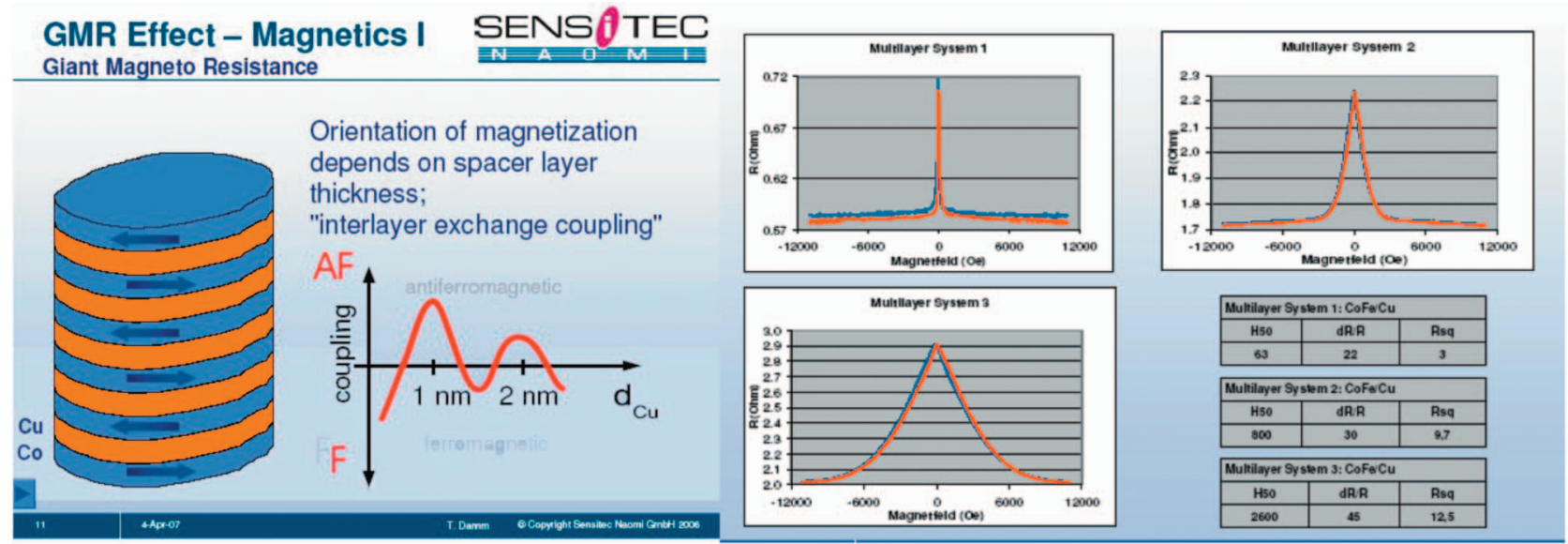

FIG. 13. (Color) Working principle and data for GMR sensor with AF-coupled multilayer (courtesy of NAOMI-Sensitech, Germany).

partly an interface effect (note that in Fig. 10 the scattering events have been intentionally placed at the interfaces), one can make GMR sensors thinner than those based on AMR. A thinner layer is subject to less demagnetizing effects than a thicker one when one also wants to shrink the lateral dimensions in order to read a narrower track. In other fields where a small size is not as critical, AMR and GMR might still coexist for some time. It is expected that GMR will save costs in the long run, alone from the trivial fact that one can have more sensors on a wafer during production.

There are two basic concepts for GMR-type sensors, which are connected with the two ways to obtain antiparallel alignment discussed in Figs. 9 and 10(b). For the sensor material in Fig. 13(a), $\mathrm{Co} / \mathrm{Cu}$ multilayer was used, where the $\mathrm{Cu}$ thickness was chosen to provide antiferromagnetic coupling. Its strength and the associated properties can be chosen from the empirical coupling curve, which is also displayed. Three different panels and a table are displayed which present these properties,

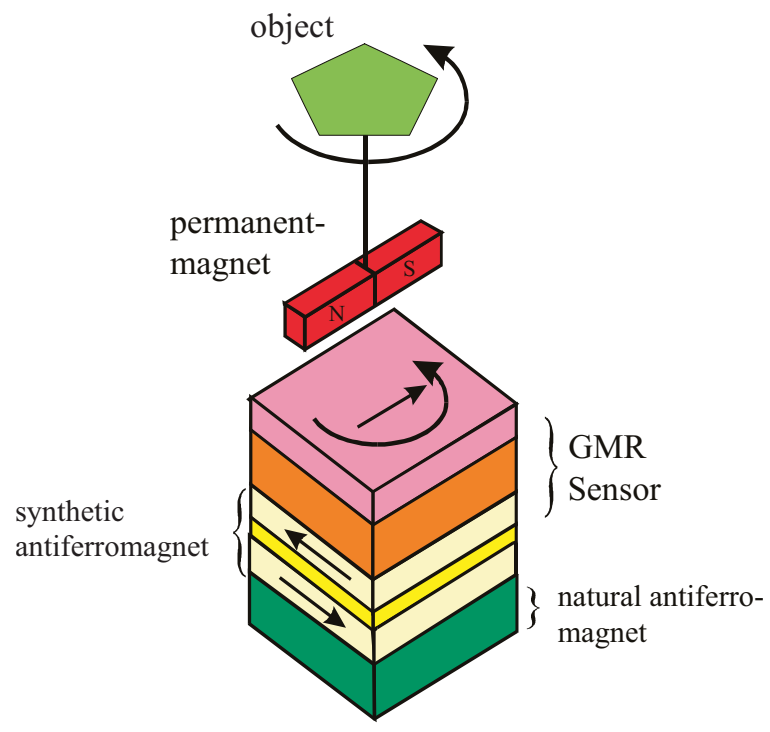

FIG. 14. (Color) Working principle of a GMR spin-valve-type sensor, used here for measurement of rotational angles. in particular saturation field and sensitivity. This type of sensor has the advantage of a large signal because multilayers can be used, but the disadvantage that it is unipolar (positive and negative fields yield the same signal as also displayed by the curves). They cannot be used for magnetic recording where the polarity of the field contains the essential information. However, there are many other applications in position and motion sensing of objects, which are magnetic or marked by attaching a little permanent magnet to them. They range from tooth detection of rotating gear wheels to the detection of cars or even airplanes for traffic control. In future car parks, each lot will be equipped with a sensor and will give information regarding free lots to the customer via a panel at the entrance.

The other type of GMR sensor also available on the market is of the spin-valve type (see above). It consists of a fixed layer (see Fig. 14) and a free layer, which can follow the applied field. There are various ways to stabilize the fixed layer, like the use of "synthetic antiferromagnets" with compensated overall magnetization such that they do not react to the applied field. They can be stabilized further using natural antiferromagnets and the well-known effect of "exchange anisotropy." The disadvantage here is that multilayers yield no improvement. GMR signals are hardly more than $10 \%$. However the real advantage is that they are bipolar and thus can be used in magnetic recording and electronic compasses.

Applications of antiferromagnetic coupling have already been indicated (synthetic antiferromagnets). This would be for use in sensors. However, magnetic storage media of HDDs have also benefited from the invention of AFC (antiferromagnetically coupled) media with improved stability of stored information.

\section{REFERENCES}

Barnás, J., A. Fuss, R. E. Camley, P. Grünberg, and W. Zinn, 1990, "Novel magnetoresistance effect in layered magnetic structures: Theory and experiment," Phys. Rev. B 42, 8110.

Barnas, J., and P. Grünberg, 1989, "Spin waves in exchange 
coupled epitaxial double layers," J. Magn. Magn. Mater. 82, 186.

Binasch, G., P. Grünberg, F. Saurenbach, and W. Zinn, 1989, "Enhanced magnetoresistance in layered magnetic structures with antiferromagnetic interlayer exchange," Phys. Rev. B 39, 4828.

Bürgler, D. E., P. Grünberg, S. O. Demokritov, and M. T. Johnson, 2001, "Interlayer exchange coupling in layered magnetic structures," Handbook of Magnetic Materials, Vol. 13, edited by K. H. J. Buschow (review) (Elsevier, New York).

Camley, R. E., and J. Barnas, 1989, "Theory of giant magnetoresistance effects in magnetic layered structures with antiferromagnetic coupling," Phys. Rev. Lett. 63, 664.

Cochran, J. F., and J. R. Dutcher, 1988, "Calculation of Brillouin light scattering intensities from pairs of exchange coupled films," J. Appl. Phys. 64, 6092.

Fert, A., P. Grünberg, A. Barthelemy, F. Petroff, and W. Zinn, 1995, "Layered magnetic structures: Interlayer exchange coupling and giant magnetoresistance," J. Magn. Magn. Mater. 140-144, 1.

Grünberg, P., 1989, "Light scattering from spin waves in layered magnetic structures," Light Scattering in Solids $V$, edited by M. Cardona and G. Güntherodt (review), Appl. Phys. Vol. 66 (Springer, Berlin).

Grünberg, P., D. Bürgler, H. Dassow, A. Rata, and C. M. Schneider, 2007, "Spin-transfer phenomena in layered magnetic structures: Physical phenomena and materials aspects," Acta Mater. 55, 1171.

Grünberg, P., and F. Metawe, 1977, "Light scattering from bulk and surface spin waves in EuO," Phys. Rev. Lett. 39, 1561.

Grünberg, P., R. Schreiber, Y. Pang, M. B. Brodsky, and H. Sowers, 1986, "Layered magnetic structures: Evidence for antiferromagnetic coupling of Fe layers across Cr-interlayer," Phys. Rev. Lett. 57, 2442.

Ibach, H., and H. Lüth, 1995, Solid State Physics (Springer, New York).

Lehndorff, R., M. Buchmeier, D. E. Bürgler, A. Kakay, R. Hertel, and C. M. Schneider, 2007, "Asymmetric spin-transfer torque in single-crystalline $\mathrm{Fe} / \mathrm{Ag} / \mathrm{Fe}$ nanopillars," Phys. Rev. B 76, 214420.

Unguris, J., R. J. Celotta, and D. T. Pierce, 1991, “Observation of two different oscillation periods in the exchange coupling of Fe/Cr/Fe(100),” Phys. Rev. Lett. 67, 140. 